\title{
Investigating the temperature dependence of the viscosity of a non-Newtonian fluid within lithographically defined microchannels
}

\author{
Salvatore Girardo, ${ }^{\text {a) }}$ Roberto Cingolani, and Dario Pisignano \\ NNL, National Nanotechnology Laboratory of CNR-INFM, Università del Salento, \\ c/o Distretto Tecnologico ISUFI, via Arnesano, I-73100 Lecce, Italy
}

(Received 3 May 2007; accepted 30 August 2007; published online 22 October 2007)

\begin{abstract}
We present a study of the rheological phenomenology of a non-Newtonian glass former within hybrid microchannels above the vitrification region. We determined the temperature behavior of the viscosity, which is well fitted by a Vogel-Fulcher-Tamman law for shear rates between $4 \times 10^{-2}$ and $9 \times 10^{-1} \mathrm{~s}^{-1}$. The microflow viscosity was compared with previously reported conductivity data of the investigated molecular system. Our findings provide an insight into the coupling between the structural dynamics in the bulk and that within the microchannels, suggesting lithographically defined microfluidic systems as promising tools for the investigation of the rheological properties of complex liquids. (C) 2007 American Institute of Physics. [DOI: 10.1063/1.2789426]
\end{abstract}

\section{INTRODUCTION}

Amorphous materials are ubiquitous in nature and in modern technology and materials science, spanning from metallic composites to conjugated polymers. ${ }^{1,2}$ Cooling determines the slowing down of the molecular motion and consequently the dramatic increase of the liquid viscosity $(\eta)$ and relaxation time. A number of experimental techniques were employed so far in order to gain insight into the supercooled molecular dynamics. ${ }^{3-9}$ However, the rationalization of the role of the topological disorder in determining the physical properties of molecular systems under vitrification is not yet achieved, and the different proposed theoretical approaches ${ }^{10-13}$ still provide an incomplete predictive capability.

Upon approaching the glass transition temperature $T_{g}$, amorphous materials are characterized by large viscosity $\left(\cong 10^{13} \mathrm{P}\right)$, and the slowing down of the structural dynamics close to $T_{g}$ is reasonably well described by the VogelFulcher-Tamman (VFT) law, ${ }^{14}$

$$
\eta(T)=\eta_{0} \exp \frac{D T_{0}}{T-T_{0}}
$$

where $D$ is the strength parameter, $\eta_{0}$ indicates the viscosity for very high temperature, and a divergence of the viscosity and the structural relaxation time is predicted at $T_{0}$ (Vogel temperature). Many recent works indicated the existence of a crossover region well above $T_{g}\left(\geqslant 1.2 T_{g}\right)$, where a number of phenomena priming the glass transition occur. These include changes in structural dynamics, ${ }^{15}$ splitting between the main and secondary relaxations, ${ }^{16}$ increase of the intermolecular cooperative coupling, ${ }^{17}$ and failure of the coupling between translational and rotational dynamics established by the Debye-Stokes-Einstein (DSE) relations. ${ }^{16,18}$ According to the DSE model, $\eta$ and the conductivity $(\sigma)$ should be related by $\sigma \eta=n_{i} e^{2} / 6 \pi r=$ const, where $n_{i}, e$, and $r$ are the density, the

\footnotetext{
${ }^{a)}$ Electronic mail: salvatore.girardo@unile.it
}

charge, and the hydrodynamic radius of the charge carrier particles in the liquid.

The rheology of polymeric liquids and organics near solid walls, with thickness ranging from tens of nanometers to tens of micrometers, is also important for the development of many applications, such as coatings, nano- and microtechnologies involving solid-fluid interfaces, spin coating of films for lithographies and plastic optoelectronics, ${ }^{19}$ and micronanofluidic devices. ${ }^{20}$ In particular, microfluidics can open new perspectives in the basic study of fluids since the penetration motion in microscale capillaries is strictly related to the rheology of the filling liquid, and microchannel devices operate with small volumes (picoliters to microliters) of chemicals and low costs. Significant advancements have been made on lab-on-chip technology, whereas rare attempts have been devoted to fundamental understanding of such fluid motion. Analytical chips can allow one to investigate glass formers under laminar conditions and high surface to volume ratio, and the microchannels can be engineered (patterned, made more polar, ${ }^{21}$ etc.) to explore different boundary conditions for the flow. For instance, many surfaces, especially hydrophobic, can determine slip (i.e., a nonzero slip length ${ }^{22}$ at the solid-liquid interface, with an enhancement of the flow rate. ${ }^{23}$

In general, the remarkable variation of the fluid viscosity upon varying temperature renders glass formers very suitable for the observation of the fluid motion within lithographically defined microchannels since the rate of penetration increases upon decreasing the fluid viscosity. Fragile compounds, which are characterized by small values of $D$, are particularly interesting for temperature-resolved microfluidic measurements, since showing a more evident slowing of their dynamics upon decreasing temperature.

Within microchannels, the shear-rate $(\dot{\gamma})$ behavior of liquids has also to be taken into account. Unlike Newtonian fluids, which are characterized by a linear relation between shear stress $(\tau)$ and $\dot{\gamma}(\tau=\eta \dot{\gamma})$, a lot of bio-organic ${ }^{24}$ and polymeric compounds exhibit nonlinear mechanical proper- 


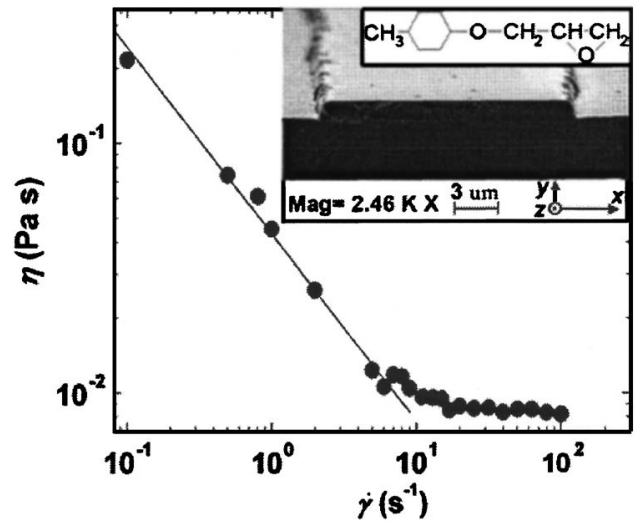

FIG. 1. CGE viscosity dependence on the shear rate, by classical rheometry at $293 \mathrm{~K}$. The line is a fit by Eq. (2) for $\dot{\gamma}<10 \mathrm{~s}^{-1}$. Inset: scanning electron micrograph of the cross section of the employed master, with scheme of the employed coordinate system, and molecular structure of CGE.

ties and peculiar viscoelastic behavior. ${ }^{25}$ In the case of nonNewtonian systems, microfluidics can be particularly useful since the capillary motion directly probes the fluid viscosity and allows one to investigate a full range of $\dot{\gamma}$ in a single penetration run into microchannels.

In this study, we applied microfluidics for the investigation of the rheological properties of a non-Newtonian glass former, i.e., the cresyl glycidyl ether (CGE), and the temperature dependence of the viscosity in the range $1.4-1.6 T_{g}$. The temperature behavior is well fitted by a VFT law, whose structural parameters were compared with previously reported conductivity data of CGE. Our findings, indicating a DSE coupling between the bulk conductivity and the viscosity of the non-Newtonian fluid under laminar flow, suggest lithographically defined microfluidic systems as promising tools for the investigation of the rheological properties of complex liquids.

\section{EXPERIMENTAL SECTION}

CGE (molecular weight of about 164, inset of Fig. 1) was purchased from Sigma-Aldrich and used as received. The compound, which is in liquid form at room temperature, was used as received, without dilution in any solvent. Epoxy compounds such as CGE revealed to be especially suitable for dielectric spectroscopy, ${ }^{16,26,27}$ by virtue of the large dielectric susceptibility, permitting accurate impedance measurements. In addition, they get a weak frequencyindependent conductivity by ionic impurities, allowing one to study the coupling between translational and rotational diffusion $^{16,25}$ and they often possess also a large optic susceptibility, thus being very suitable to compare results obtained by different experimental approaches. ${ }^{28}$

The microchannels of our assembled device, of height $(h) \cong 1.2 \mu \mathrm{m}$, width $\cong 17.0 \mu \mathrm{m}$, and length $(L) \cong 1 \mathrm{~cm}$, were realized by the contact superposition of a polydimethylsiloxane (PDMS) element, negatively replicating a template realized by optical lithography (cross section in the inset of Fig. 1), and a substrate of $\mathrm{Si}-\mathrm{SiO}_{2} \cdot{ }^{29}$ The elastomeric replicas were obtained by the in situ polymerization $\left(140{ }^{\circ} \mathrm{C}\right.$ for $15 \mathrm{~min}$ ) of PDMS (Sylgard 184 by Dow Corn- ing, Midland, MI, $A: B$ 1:9) according to a standard replica molding procedure.

An integrated ruler was fabricated side by side with the planar capillary, allowing us to accurately measure the microfluidic filling rate by optical microscopy. ${ }^{30}$ The system temperature was varied between 293 and $333 \mathrm{~K}$, avoiding thermal gradients during measurements. We could not observe any evaporation phenomena during measurements.

In addition, rheological measurements were carried out by a rotational rheometer (TA Instruments Inc.) equipped with a dual range rebalance transducer. A Couette geometry with bob and cup sizes of, respectively, 34 and $32 \mathrm{~mm}$ filled with $15 \mathrm{ml}$ of sample volume was employed, with shear rates between $10^{-1}$ and $10^{2} \mathrm{~s}^{-1}$.

\section{RESULTS AND DISCUSSION}

The coordinate, $z$, of the liquid front along the microchannel increases during the capillary penetration according to the temporal dependence, $z=A t^{\beta}$, where one usually employs $\beta=0.5$ (Washburn equation) $^{31}$ and $A \approx(P / \eta)^{1 / 2}$, $P$ being the effective pressure difference driving the capillary motion. Though a simplified Newtonian $(\beta=0.5)$ description is largely adopted for the lithographic applications of microfluidics, ${ }^{31-33}$ experimental findings for organics in microchannels often exhibit remarkably different behaviors with the consequence of an effectively halted capillary flow. ${ }^{32}$ The CGE viscosity dependence on the shear rate at room temperature, determined by rotational rheometry, is displayed in Fig. 1, clearly indicating a roughly Newtonian and a non-Newtonian behavior for $\dot{\gamma}>10 \mathrm{~s}^{-1}$ and for $\dot{\gamma}<10 \mathrm{~s}^{-1}$, respectively. The non-Newtonian region can be effectively described by a model by Ostwald, ${ }^{34}$

$$
\eta=m \dot{\gamma}^{n-1},
$$

where $m\left(\mathrm{~Pa} s^{n}\right)$ is the flow consistency coefficient and $n$ (dimensionless) is a flow behavior index (when $n=1$ and $m=\eta$, a Newtonian representation is recovered). Fitting the rheometry data collected for $\dot{\gamma}<10 \mathrm{~s}^{-1}$ provided a value $n=(0.25 \pm 0.03)$ (Fig. 1).

In microchannels, the fluid velocity is described by Navier-Stokes equations ${ }^{35,36}$ that, using the Ostwald-de Waele model, for incompressible fluids can be expressed, in the simplified case of a cylindrical capillary with radial coordinate $r$, as $d P / d z=(1 / r)(\partial / \partial r)\left[m r\left(d u_{z} / d r\right)^{n}\right]$, where $u_{z}$ indicates the $z$ component of the fluid velocity in microchannels. The previous equation, solved under the assumptions of neglectable linear convective acceleration and dominating velocity gradients in the $y$ direction (cross section in Fig. 1), finally gives

$$
z=G\left(\frac{P}{m}\right)^{1 /(n+1)} t^{n /(n+1)}=A t^{n /(n+1)},
$$

where $G$ depends on the geometry of the channel and on $n$, $P$ is the pressure drop throughout $L,{ }^{32}$ and the phenomenological constant $A$ depends on the system temperature and on eventual nonzero slip lengths.

For each temperature, experimental $(z, t)$ data were fitted by Eq. (3), namely, by a law with two fitting parameters, 


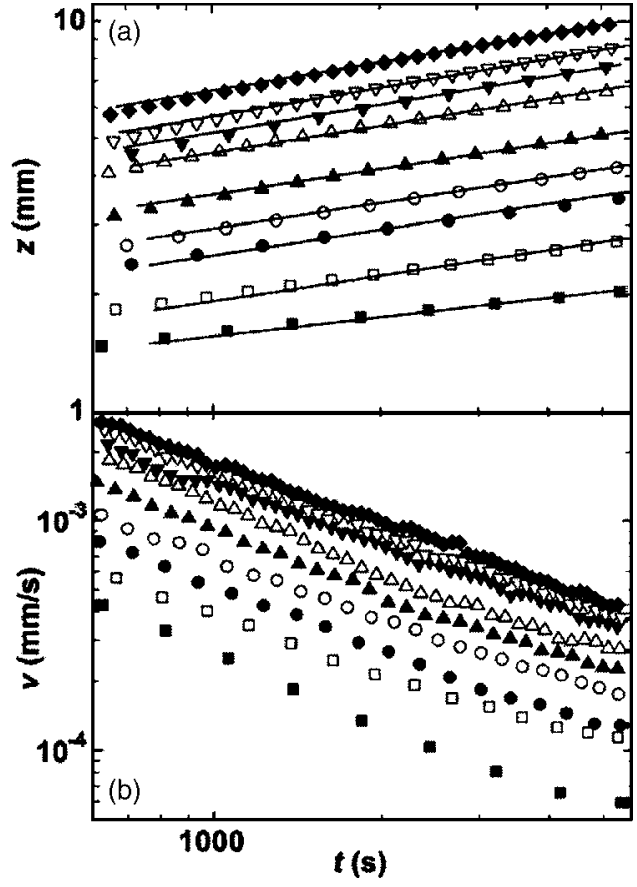

FIG. 2. (a) Covered length $z$ vs time $t$ at different temperatures, with the best fit according to Eq. (3). The experimental errors are smaller than employed symbols. (b) Filling rate $\dot{z}$ vs time $t$ at different temperatures. (ם) $293 \mathrm{~K},(\square) 298 \mathrm{~K},(\bullet) 303 \mathrm{~K},(\bigcirc) 307 \mathrm{~K},(\Delta) 314 \mathrm{~K},(\triangle) 318 \mathrm{~K},(\nabla)$ $324 \mathrm{~K},(\nabla) 329 \mathrm{~K}$, and $(\diamond) 333 \mathrm{~K}$.

$A$ and $n$. The experimental data together with the best fitting curves, and the $(\dot{z}, t)$ data found for CGE at temperatures between 293 and $333 \mathrm{~K}$, are shown in Figs. 2(a) and 2(b), respectively. The liquid fills completely the microchannels $(L=10 \mathrm{~mm})$ in $5400 \mathrm{~s}$ at $333 \mathrm{~K}$, with velocity decreasing down to $0.4 \mu \mathrm{m} / \mathrm{s}$ along the capillary. The glass transition behavior of the monoepoxy directly affects the capillary motion because of the abrupt increase of the fluid viscosity upon decreasing temperature, determining a rapid decrease of the filling rate. At $293 \mathrm{~K}$, the fluid penetrates into the channels until a maximum value, $z$ about $2.0 \mathrm{~mm}$, in a time as large as $5400 \mathrm{~s}$. Upon filling, the penetration velocity decreases to less than $0.06 \mu \mathrm{m} / \mathrm{s}$. Therefore, a temperature reduction of about $40 \mathrm{~K}$ induces a decrease of almost seven times of the residual penetration speed at the end of the experimental observation. Overall, the microfluidic data of CGE are well described by Eq. (3), with the exponent $\beta=n / n+1=0.22 \pm 0.03$, i.e., $n=0.28 \pm 0.05$ (Fig. 2), in agreement with the value of the flow behavior index obtained by classical rheometry.

Our analysis aimed then at evidencing the temperature dependence of the penetration dynamics. This is implicit in Eq. (3) and mainly due to viscosity, on its turn connected to the flow consistency coefficient by Eq. (2). In the following, the weak dependence of $n$ on temperature ${ }^{35}$ will be neglected with respect to exponential-like (VFT) behaviors, such as that exhibited by $\eta$ in the supercooled regime. Considering $\dot{\gamma} \approx \dot{z} / h{ }^{37}$ we obtain for the penetrating CGE a relation between the capillary length and the shear rate,

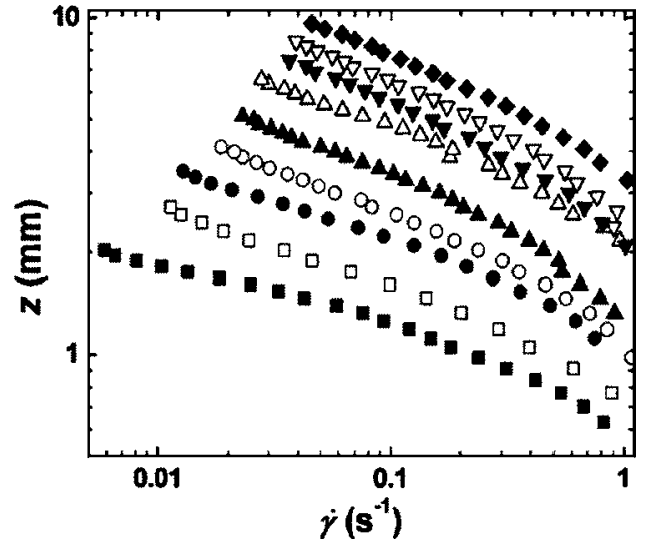

FIG. 3. Covered length $z$ vs shear rate $\dot{\gamma}$ at different temperatures. The values of $\dot{\gamma}$ are between $4 \times 10^{-2}$ and $9 \times 10^{-1} \mathrm{~s}^{-1}$. Same symbols as those used in Fig. 2.

$$
z=\left(\frac{n}{n+1}\right)^{n} \frac{G^{n+1} P}{m h^{n}} \frac{1}{\dot{\gamma}^{n}}
$$

The measured $(z, \dot{\gamma})$ curves are displayed in Fig. 3. To extract the dependence of the CGE viscosity on temperature, we first determined the ratios, $z\left(\dot{\gamma}, T_{\mathrm{RT}}\right) / z\left(\dot{\gamma}, T_{i}\right)$, that, for each value of the shear rate and for each temperature value, $T_{i}$, directly provide the quantity $\eta\left(\dot{\gamma}, T_{i}\right) / \eta\left(\dot{\gamma}, T_{\mathrm{RT}}\right)$, by Eqs. (2) and (4). The viscosity curves were then normalized by the value of the room temperature viscosity at $\dot{\gamma}=1 \mathrm{~s}^{-1}$, which was measured to be $4.5 \times 10^{-2} \mathrm{Pas}$ (Fig. 1). This procedure allowed us to obtain the curves, $\eta(\dot{\gamma})$, of CGE within the
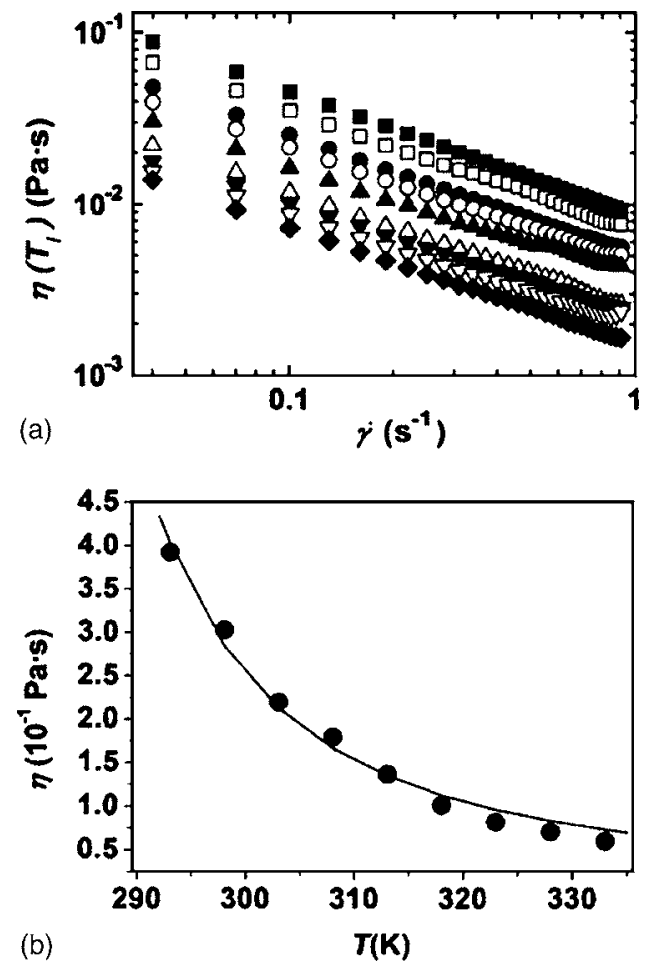

FIG. 4. (a) Viscosity vs shear rate $\dot{\gamma}$ at different temperatures. Same symbols as those used in Fig. 2(b) Temperature dependence of the viscosity of CGE in microchannels for $\dot{\gamma}=0.05 \mathrm{~s}^{-1}$. The superimposed line is the VFT law with parameters $D=0.77 \pm 0.05$ and $T_{0}=244 \pm 3 \mathrm{~K}$. 
microfluidic device at different temperatures [Fig. 4(a)]. For each value of $\dot{\gamma}$, the viscosity decreases upon increasing temperature, for instance, ranging from $4 \times 10^{-1} \mathrm{Pas}$ (at $T=293 \mathrm{~K}$ ) to $6 \times 10^{-2} \mathrm{~Pa} \mathrm{~s}$ (at $T=333 \mathrm{~K}$ ) for $\dot{\gamma}=5 \times 10^{-2} \mathrm{~s}^{-1}$. The corresponding change rate $(d \eta / d T)$ is large for low temperature, progressively decreasing when temperature increases [Fig. 4(b)]. This behavior is well reproduced by a VFT law with parameters $D=0.77 \pm 0.05$ and $T_{0}=244 \pm 3 \mathrm{~K}$ [fitting curve in Fig. 4(b)]. The low value of $D$ indicates CGE as a fragile glass-former compound. The value of viscosity has been calculated for different shear rates $\left(4 \times 10^{-2} \mathrm{~s}^{-1} \leqslant \dot{\gamma} \leqslant 9 \times 10^{-1} \mathrm{~s}^{-1}\right)$, providing similar VFT parameters.

The glass transition dynamics of CGE was investigated by Corezzi et $a .^{26}$ by dielectric spectroscopy. Our results agree with their findings about $T_{0}$ and $D$, obtained for the structural dynamics in the high-temperature regime by conductivity $(\sigma)$ data. This result indicates that a DSE coupling occurs between the conductivity and the viscosity of the nonNewtonian fluid under laminar flow, namely, the monoepoxy within the microfluidic device shows a temperature dynamics for translational diffusion processes comparable to that measured by dielectric spectroscopy in the bulk material. We point out that, in the investigated range of temperatures $\left(>1.4 T_{g}\right)$, the conductivity-viscosity coupling would be expected in the bulk material. In our case, this finding provides an insight into the coupling between the structural dynamics in the bulk and that within the restricted geometry of the microchannel environment. This result is physically reasonable since the influence of confinement on the mobility and the structural dynamics of molecular systems is generally relevant for system lengths below the scale of $100 \mathrm{~nm},{ }^{19,38}$ that is, around one order of magnitude below the smallest dimension of our microchannels. Therefore, a proper choice of the fluidic geometry allows one to employ very minute amount of sample, concomitantly retaining the condition of bulk materials. In the future, fabrication technologies providing higher resolutions, such as soft lithography using hard elastomeric materials and optimized etching and lift-off procedures, will be employed to fabricate fluidic circuits with vertical $(y)$ dimension of the order of a few tens of nanometers, retaining micrometer size in the horizontal $(x)$ direction. These will enable the optical observation of complex fluids in nanofluidic geometries and the study of the dimensional dependence of their structural dynamics and glass transition phenomenology.

\section{CONCLUSION}

In summary, we investigated the rheological behavior of the non-Newtonian glass-former CGE within lithographically defined microchannels in the range $1.4-1.6 T_{g}$. We described the penetration dynamics in terms of an Ostwald-de Waele model for the viscosity dependence on the liquid shear rate. The viscosity could be measured by the $(z, t)$ and $(\dot{z}, t)$ filling curves of the hybrid microchannels. For each value of $\dot{\gamma}$, the temperature dependence of $\eta$ can be described by a VFT law, whose strength parameter and Vogel temperature are in agreement with conductivity data ${ }^{26}$ for the same tem- perature range. This study suggests lithographically defined microfluidic systems as promising tools for the investigation of the rheological properties of complex liquids.

\section{ACKNOWLEDGMENTS}

This work was partially supported by the Italian Minister of Education, University and Research through the FIRB project "Laboratorio nazionale sulle nanotecnologie per la genomica e postgenomica" (NG-Lab).

${ }^{1}$ P. G. Debenedetti and F. H. Stillinger, Nature (London) 410, 259 (2001).

${ }^{2}$ C. A. Angell, K. L. Ngai, G. B. McKenna, P. F. McMillan, and S. W. Martin, J. Appl. Phys. 88, 3113 (2000).

${ }^{3}$ F. Garwe, A. Schönhals, M. Beiner, K. Schröter, and E. Donth, J. Phys.: Condens. Matter 6, 6941 (1994).

${ }^{4}$ D. Fioretto, A. Livi, P. A. Rolla, G. Socino, and L. Verdini, J. Phys.: Condens. Matter 6, 5295 (1994).

${ }^{5}$ A. Arbe, D. Richter, J. Colmenero, and B. Farango, Phys. Rev. E 54, 3853 (1996).

${ }^{6}$ G. F. Floudas, J. S. Higgins, F. Kremer, and E. W. Fischer, Macromolecules 25, 4955 (1992).

${ }^{7}$ J. F. Shi, P. T. Inglefield, A. A. Jones, and M. D. Meadows, Macromolecules 29, 605 (1996).

${ }^{8}$ F. Garwe, M. Beiner, E. Henpel, J. Schawe, A. Schröter, and E. Donth, J. Non-Cryst. Solids 172-174, 191 (1994).

${ }^{9}$ M. Reihhardt, A. Schönhals, K. Pteiffer, I. V. Lampe, and H. J. Lorkowski, Polym. Adv. Technol. 7, 791 (1996).

${ }^{10}$ M. H. Cohen and D. Turnbull, J. Chem. Phys. 31, 1164 (1959).

${ }^{11}$ G. Adam and J. H. Gibbs, J. Chem. Phys. 43, 139 (1965).

${ }^{12}$ K. L. Ngai, J. Chem. Phys. 111, 3639 (1999).

${ }^{13}$ W. Götze and L. Sjögren, Rep. Prog. Phys. 55, 242 (1992).

${ }^{14}$ H. Vogel, Phys. Z. 22, 645 (1921); G. S. Fulcher, J. Am. Ceram. Soc. 8, 339 (1925); G. Tamman and W. Hesse, Z. Anorg. Allg. Chem. 156, 245 (1926).

${ }^{15}$ F. Stickel, E. W. Fischer, and R. Richert, J. Chem. Phys. 102, 6251 (1995).

${ }^{16}$ S. Corezzi, E. Campani, P. A. Rolla, S. Capaccioli, and D. Fioretto, J. Chem. Phys. 111, 9343 (1999).

${ }^{17}$ R. Casalini, K. L. Ngai, and C. M. Roland, Phys. Rev. B 68, 014201 (2003).

${ }^{18}$ E. Rössler, Phys. Rev. Lett. 65, 1595 (1990).

${ }^{19}$ S. Sills, R. M. Overney, W. Chau, V. Y. Lee, R. D. Miller, and J. Frommer, J. Chem. Phys. 120, 5334 (2004).

${ }^{20}$ T. M. Squires and S. R. Quake, Rev. Mod. Phys. 77, 977 (2005).

${ }^{21}$ D. Pisignano, F. Di Benedetto, L. Persano, G. Gigli, and R. Cingolani, Langmuir 20, 4802 (2004).

${ }^{22}$ S. Granick, Y. Zhu, and H. Lee, Nat. Mater. 2, 221 (2003).

${ }^{23}$ E. Lauga, Langmuir 20, 8924 (2004).

${ }^{24}$ S. Chakraborty, Lab Chip 5, 421 (2005).

${ }^{25}$ A. Groisman and V. Steinberg, Nature (London) 53, 405 (2000).

${ }^{26}$ S. Corezzi, S. Capaccioli, G. Gallone, A. Livi, and P. A. Rolla, J. Phys.: Condens. Matter 9, 6199 (1997).

${ }^{27}$ S. Corezzi, D. Fioretto, and P. A. Rolla, Nature (London) 420, 653 (2002).

${ }^{28}$ L. Comez, D. Fioretto, L. Verdini, and P. A. Rolla, J. Phys.: Condens. Matter 9, 3973 (1997); D. Fioretto, L. Comez, G. Socino, L. Verdini, S. Corezzi, and P. A. Rolla, Phys. Rev. E 59, 1899 (1999).

${ }^{29}$ D. Pisignano, E. Sariconi, M. Mazzeo, G. Gigli, and R. Cingolani, Adv. Mater. (Weinheim, Ger.) 14, 1565 (2002).

${ }^{30}$ An estimate of the experimental error associated with the measured $z$ data is provided by the ratio between the fluid velocity and the acquisition frame rate of the camera connected to the microscope (30 frames/s). The maximum measured velocity of the CGE front into microchannels was of the order of $10^{2} \mu \mathrm{m} / \mathrm{s}$, corresponding to an experimental error for the evaluation of the fluid position of about $3 \mu \mathrm{m}$. Of course, the lower limit for the achievable precision is given by the spatial resolution of the employed optical microscope $(\sim 1 \mu \mathrm{m})$.

${ }^{31}$ E. W. Washburn, Phys. Rev. 17, 273 (1921).

${ }^{32}$ E. Delamarche, A. Bernard, H. Schmid, A. Bietsch, B. Michel, and H. Biebuyck, J. Am. Chem. Soc. 120, 500 (1998).

${ }^{33}$ N. L. Jeon, I. S. Choi, B. Xu, and G. M. Whitesides, Adv. Mater. (Wein- 
heim, Ger.) 11, 946 (1999).

${ }^{34}$ W. Ostwald, Kolloid-Z. 36, 99 (1925); A. de Waele, Journal of Oil and Colour Chemists Association 6, 33 (1923).

${ }^{35}$ R. B. Bird, C. F. Curtiss, and R. C. Armstrong, Dynamic of Polymeric Liquids (Wiley, New York, 1960).
${ }^{36}$ D. J. Tritton, Physical Fluid Dynamics (Clarendon, Oxford, 1987).

${ }^{37}$ N. Srivastava and M. A. Burns, Anal. Chem. 78, 1690 (2006).

${ }^{38}$ X. Zheng, M. H. Rafailovich, J. Sokolov, Y. Strzhemechny, S. A. Schwarz, B. B. Sauer, and M. Rubinstein, Phys. Rev. Lett. 79, 241 (1997). 
The Journal of Chemical Physics is copyrighted by the American Institute of Physics (AIP). Redistribution of journal material is subject to the AIP online journal license and/or AIP copyright. For more information, see http://ojps.aip.org/jcpo/jcper/jsp 